Review

\title{
Targeting autophagy as a potential therapeutic approach for melanoma therapy
}

He Liu, Zhaoyue He, Hans-Uwe Simon*

Institute of Pharmacology, University of Bern, Friedbuehlstrasse 49, CH-3010 Bern, Switzerland.

\begin{abstract}
Melanoma, occurring as a rapidly progressive skin cancer, is resistant to current chemoand radiotherapy, especially after metastases to distant organs has taken place. Most chemotherapeutic drugs exert their cytotoxic effect by inducing apoptosis, which, however, is often deficient in cancer cells. Thus, it is appropriate to attempt the targeting of alternative pathways, which regulate cellular viability. Recent studies of autophagy, a well-conserved cellular catabolic process, promise to improve the therapeutic outcome in melanoma patients. Although a dual role for autophagy in cancer therapy has been reported, both protecting against and promoting cell death, the potential for using autophagy in cancer therapy seems to be promising. Here, we review the recent literature on the role of autophagy in melanoma with respect to the expression of autophagic markers, the involvement of autophagy in chemo- and immunotherapy, as well as the role of autophagy in hypoxia and altered metabolic pathways employed for melanoma therapy.
\end{abstract}

*Corresponding author. Tel.: +41 31632 3281; fax: +41 316324992.

E-mail address: hus@pki.unibe.ch (H.U. Simon) 


\section{Keywords:}

\section{Autophagy}

Chemotherapy

Hypoxia

Immunotherapy

Melanoma

Abbreviations:

5-ALA, 5-aminolevulinic acid; AMBRA1, autophagy/Beclin 1 regulator 1; APC, antigen-presenting cell; ASS, argininosuccinate synthetase; ATG, autophagy-related; ATM, Ataxia telangiectasia mutated: BafA1, bafilomycin A1; Bif-1, endophilin B1; BN, benign nevi; Cav-1, caveolin-1; CQ, chloroquine; CTL, cytolytic T lymphocyte; CTLA-4, cytotoxic T-lymphocyte antigen 4; DDR, DNA damage response; DN, dysplastic nevi; DRiPs, defective ribosomal products; EM, electron microscopy; ER, endoplasmic reticulum; GA-DM, ganoderic acid-dextromethorphan; HIF, hypoxia-inducible factor; HLA, human leucocyte antigen; IFN, interferon; LAMP2, lysosomal-associated membrane protein 2; LC3, microtubuleassociated protein 1 light chain 3; 3-MA, 3-methyladenine; MHC, major histocompatibility complex; mTOR, mammalian target of rapamycin; PCL, polygonatum cyrtonema lectin; PE, phosphatidylethanolamine; PEDF, pigment epithelium-derived factor receptor; PEI, polyethylene imine; PI3K, phosphoinositide 3-kinase; PFS, progression free survival; PI3P, phophatidylinositol-3-phosphate; RGP, radial growth phase; ROS, reactive oxygen species; SKP, skin-derived precursor; TMA, tissue microarray; TRAIL, tumor necrosis factor-related apoptosis-inducing ligand; ULK1, unc-51-like kinase 1; UVRAG, UV radiation resistance associated gene; VGP, vertical growth phase 


\section{Introduction}

Autophagy is an evolutionally conserved cellular "self-eating" process through which cells digest their own cellular contents via the lysosomal machinery, thereby providing energy and building blocks for cell survival. Autophagy is not a static process, rather a dynamic one during which long-lived proteins and/or organelles are engulfed in a doublemembrane autophagosome, which then fuses with lysosomes or late endosomes in order to degrade the sequestered contents with lysosomal enzymes [1] (Fig. 1).

Three major types of autophagy have been reported in mammalian cells, including macroautophagy, microautophagy, and chaperon-mediated autophagy. These types differ in the cytosolic targets to be degraded and in the involvement of lysosomes. Macro- and microautophagy sequester cellular contents, e.g. organelles, whereas chaperon-mediated autophagy targets only soluble, long-lived proteins. The topic of this review is macroautophagy, hereafter referred to just as autophagy. During macroautophagy, cellular elements are engulfed by an isolating membrane of nonlysosomal origin, probably originating in endoplasmic reticulum (ER) $[2,3]$, which expands and seals to form autophagosomes. Fusion of autophagosomes with lysosomes facilitates the degradation by lysosomal enzymes [4]. According to the organelle being degraded, autophagy can be further divided into mitophagy (selective for mitochondria), pexophagy (selective for peroxisomes), reticulophagy (selective for ER), and so on $[5]$.

Autophagy is a highly regulated process, in which the activities of autophagy-related (ATG) proteins are involved. They can be divided into 4 functional groups. ATG proteins which regulate the induction of autophagy include ATG1 or ULK1, ATG13 and ATG17. ATG proteins responsible for vesicle nucleation include ATG6 or Beclin 1 and ATG9. Other ATG proteins are involved in autophagosome formation, such as ATG5, ATG12 and ATG8 or LC3 (Fig. 1). And, finally, there are ATG proteins responsible for the retrieval of autophagy related proteins that include ATG2, ATG9 and ATG18 [6-9].

Besides ATG proteins, autophagy is also controlled by mammalian target of rapamycin (mTOR) and class I and class III phosphoinositide 3-kinase (PI3K) pathways. mTOR and class I PI3K act to inhibit autophagy, whereas the class III PI3K complex 
induces autophagy. Therefore, pharmacological inhibitors of mTOR, e.g. rapamycin, activate autophagy (Fig. 1) [6]. A dual effect of 3-MA on autophagy has been shown since its persistent blockage of class I PI3K induces autophagy, while a transient suppression of class III PI3K inhibits autophagy $[10]$. Detailed descriptions of the molecules and/or pathways regulating autophagy have been reviewed elsewhere [11, 12].

Even if autophagy is primarily understood as a pro-survival mechanism, interactions between the regulators involved in autophagy and in apoptosis have been described. ATG5, a key regulator of autophagy required for autophagosome formation, has also been shown to be cleaved by calpain, translocated to the mitochondria, and to induce apoptosis $[13]$. Bcl-2, an anti-apoptotic protein, is shown also to interact with Beclin 1, thereby inhibiting autophagy [14]. LC3 has been shown to be involved in cigarette smoke-induced apoptosis in mouse lung epithelia through interaction with Cav-1 and FAS [15].

Autophagy is induced by cellular stress factors such as nutrient deprivation, growth factor withdrawal, and hypoxia $[16]$, though it maintains a basal level under normal physiological conditions in cells. Autophagy regulates cellular homeostasis by maintaining a balance of synthesis and degradation. It has also been found to be involved in development and differentiation [17], aging [18] and immunity [11]. Both insufficient and excessive autophagy can lead to pathological conditions. Dysregulated autophagy has been implicated in diverse diseases, including infection $[11,19]$, neurodegenerative diseases $[20,21]$, cardiovascular diseases $[22,23]$, metabolic diseases [24-26], pulmonary diseases [27-29], and cancer [30-32].

Emerging evidence indicates a context-dependent role of autophagy in cancer. On the one hand, autophagy has a tumor suppressive function by inhibiting the proliferation of precancerous cells $[33,34]$, by removing damaged organelles, e.g. mitochondria, thereby decreasing the risk of genome instability $[30]$ and by inducing senescence [35]. On the other hand, autophagy may promote tumor progression by providing 
tumor cells with nutrients under the conditions of nutrient- and oxygen-shortage, typical of the tumor environment $[4]$. Direct evidence linking autophagy to tumor suppression comes from the fact that certain ATG proteins, such as Beclin 1, UVRAG, and Bif-1, exhibit an anti-oncogenic function. Beclin 1, as the first ATG to be identified as a tumor suppressor, has been found to be monoallelically deleted in breast, ovarian and prostate tumors [36-38]. An interaction partner of Beclin 1, UVRAG induces autophagy, thereby suppressing proliferation and tumorigenicity of colon cancer cells [39]. Bif-1, which has been found to join in the Beclin 1-UVRAG complex, prevents spontaneous tumor formation in a mouse model [40]. Furthermore, deletion of atg5 in mouse liver resulted in spontaneous tumor formation [41]. Indirectly, oncogenic pathways often show an inhibitory effect on autophagy, e.g. activated PI3K, whereas tumor suppressor genes frequently have the capability to induce autophagy, e.g. PTEN [42, 43] .

In established tumors, however, autophagy is believed mainly to protect tumors cells from drug-induced stresses, thus promoting drug resistance. Here, we review recent publications, which seem to unveil a role for autophagy in melanoma cancer therapy.

\section{The expression of ATG proteins in melanoma specimens}

Preclinical studies have implicated a potential tumor suppressive function of autophagy in the initiation of tumor formation, but a protective role favoring tumor cell survival once the tumor has already formed $[30]$. Due to the limitations of techniques for monitoring the autophagy status in vivo, the results about autophagy levels in tumors have been mainly elucidated with in vitro experiments: e.g. using cell lines overexpressing GFP-LC3 to monitor autophagosomes or detecting p62 degradation and LC3 lipidation via Western Blot to monitor autophagic flux [44]. For developing small molecules targeting autophagy for clinical application, the importance of evaluating autophagy levels in patients is self-evident. Several, although not many publications, have described the level of autophagy in melanoma tumor specimens using immunostaining techniques to detect the expression of ATG proteins. However, the results obtained in these studies have varied, probably owing to the heterogeneity of the 
melanoma tumors themselves and to the different staining protocols employed for assessing the level of autophagy.

In 13 cases of superficially spreading melanomas, LC3 immunostaining (using a polyclonal antibody against LC3B from Abcam) exhibited a heterogeneous vesicular pattern in the cytoplasm, whereas normal melanocytes were only weakly LC3-positive [45]. In contrast, Miracco et al. described a moderate cytoplasmic staining of LC3 in normal melanocytes and benign nevi (BN). Additionally, they observed a heterogeneous LC3 expression pattern in melanomas, including radial growth phase (RGP), vertical growth phase (VGP), and metastatic melanomas, using an LC3B antibody obtained from Sigma $[46]$. A large scale of study of autophagy in melanoma using tissue microarrays (TMA) containing primary melanomas $(n=131)$ and unmatched metastases $(n=250)$ showed a significantly higher level of LC3 expression in metastases than that in primary tumors (using the polyclonal LC3B antibody from Abcam) $[45]$. In contrast to these findings, Miracco et al. showed a moderate density of fine LC3 positive punctae, but rarely a diffuse LC3 cytoplasmic expression in normal melanocytes. However, in dysplastic nevi (DN) and melanomas, the LC3 staining pattern was rather heterogeneous, ranging from almost undetectable to diffuse cytoplasmic and finally even to large LC3positive dots (LC3B antibody from Sigma) [46] .

Besides LC3, Beclin 1 was also examined in melanoma specimens in some studies. For example, in normal melanocytes, BN and in the majority of DN, Beclin 1 exhibited a moderate-to-strong cytoplasmic staining and nucleolar positivity. In melanomas, Beclin 1 expression seemed decreased with disease progression from RGP melanomas to metastases (Beclin 1-specific antibody from Santa Cruz was used for this study) [46], suggesting a tumor suppressive function of autophagy in melanoma. Consistent with the results of Miracco et al., Hara et al. showed a high level of Beclin 1 in advanced melanoma, but a low expression in melanoma in-situ (Beclin 1 antibody from Santa Cruz), suggesting a stage-dependent character for autophagy in melanoma [47]. A link between autophagy and clinical outcome of melanoma patients has been documented by Sivridis et al. They showed a diffuse and variably cytosolic expression of LC3 (LC3A antibody from Abgent) and Beclin 1 (antibody from Abcam) in 79 cases of nodular 
melanomas. Low Beclin 1 expression was correlated with invasiveness, tumor ulceration, and a decrease in 5-year survival after surgery. Interestingly, high levels of Beclin 1 expression were found in hypoxic areas in some melanoma specimens and its expression was linked to an increase in early deaths [48]. Half of the specimens showed a strong, diffuse staining of LC3 with no focal or punctate pattern (LC3A antibody from Abgent $[46,48]$. Taken together, it seems that different LC3 antibodies, recognizing different LC3 isoforms (LC3A or LC3B), could lead to different results.

Contradicting the studies described previously, Ma et al. examined autophagosomes with electron microscopy in melanoma tumor specimens from 12 patients enrolled on a phase II trial of temozolomide and sorafenib [49]. Patients, who had a median progression free survival (PFS) of less than 2 months, had a significantly higher number of autophagosomes in their tumor specimens than those who had a median PFS of 2 or more than 2 months. Furthermore, they found no clear correlation between autophagy and BRAF or NRAS oncogene mutations in the tumors [49]. In spite of the divers or even contradictory results of these studies, a systematic and in-depth evaluation of autophagy status in melanoma seems to be called for, examining the expression of further ATG proteins in better defined patients' samples.

\section{The dual role for autophagy in melanoma cells receiving treatment with chemotherapeutic drugs}

Most of our knowledge about autophagy in melanoma came from the studies on melanoma chemotherapy in relatively large number of publications. These data were based mostly on in vitro experiments using established melanoma cell lines to monitor autophagosome formation and/or autolysosome degradation in the presence of a variety of therapeutic agents (Table 1).

Autophagy as a protective mechanism against chemotherapy-induced melanoma cell death has been reported in several recent publications. For example, Marino et al. have shown that induction of autophagy following treatment with esomeprazole, a proton pump inhibitor, delayed melanoma cell killing. Esomeprazole treatment impaired the mTOR signaling in the earlier phase, followed later by a rapid induction of ROS leading 
to cell death. Furthermore, knocking down ATG5 or Beclin 1 expression significantly increased the efficacy of esomeprazole-induced melanoma cell death, indicating a protective role of autophagy $[50]$. Similarly, sanguilutine, which induces necroptosis in melanoma cells, triggered an autophagic response. Inhibition of autophagy with 3-MA, bafilomycin A1 (BafA1) or LY294002 in combination with sanguilutine resulted in significant reductions in melanoma cell viability [51]. G-quadruplex ligand, with the capability to interfere with guanosine-rich DNA/RNA sequences such as telomeres, impairs tumor cell growth by direct interfere with the telomere architecture, but had no effect on cell viability of normal cells [52]. Interestingly, it was reported that autophagy induced by G-quadruplex ligand in melanoma cells was a consequence of drug-induced telomere uncapping, ATM-dependent DDR and transactivation of the cyclin-dependent kinase inhibitor 1A [53]. More importantly, pharmacological and genetic inhibition of autophagy intensified drug-induced cytotoxicity in melanoma cells, suggesting that autophagy as a pro-survival mechanism contributed to the resistance of melanoma cells to the G-quadruplex ligand [53]. Tumor cells are known to change their metabolism from oxidative phosphorylation to aerobic glycolysis, resulting in increased production of metabolic acids and, thereby, tumor acidosis [54]. Marino et al. investigated the response of melanoma cells under acidic culture conditions in vitro and found a rapid inhibition of the mTOR pathway, leading to an induction of autophagy. Besides, the authors showed that inhibition of autophagy after knocking down ATG5 expression reduced the survival of melanoma cells exposed to an acidic environment $[54]$.

Contrary results showing autophagy assisting cytotoxicity as a cellular response to chemotherapeutic agents have also been described in the literature. Cysteamine, a degradation product of cysteine in vivo [55], increased the autophagosome formation in the early phase of the treatment, whereas it blocked the autophagic degradation in melanoma cells in a later phase. A synergetic effect of cysteamine and doxorubicin on cell killing was observed, which was, however, reduced by knocking down ATG5 expression. Interestingly, a concentration-dependent response of melanoma cells towards doxorubicin in combination with cysteamine was observed, which, however, was absent 
in melanoma cells knocked down for ATG5 expression [56]. Similarly, Tomic et al. have shown that metformin, a widely used antidiabetic drug [57], induced cell cycle arrest after $24 \mathrm{~h}$, induced autophagy after $72 \mathrm{~h}$ and finally induced a robust apoptosis after 96h. Importantly, they showed that inhibition of autophagy caused by knocking down LC3 or ATG5 also reduced the pro-apoptotic and anti-proliferative effect of metformin, indicating that autophagy is indispensable for metformin-mediated cytotoxicity in melanoma cells [58]. Another supporting report came from the study of terfenadine, an $\mathrm{H} 1$ histamine receptor antagonist, by Nicolau-Galmés et al. They showed that terfenadine induced ROS-dependent apoptosis in the presence of serum, whereas it induced ROS-independent cell death to an even higher degree in serum-free medium. Furthermore, they showed terfenadine induced autophagy in serum-free medium and concluded that knockdown of ATG7 partially prevented terfenadine-induced cytotoxicity [59]. These data again indicate that induction of autophagy may promote melanoma cell death induced by diverse cytotoxic compounds. A ROS-dependent activation of apoptosis and autophagy was shown in a study of the cytotoxic effect of polygonatum cyrtonema lectin (PCL), a mannose-binding lectin. The authors demonstrated that PCL treatment induced ROS production and p38-p53 activation leading to induction of both apoptosis and autophagy $[60]$. But the role of autophagy in this context was not fully clarified.

Moreover, certain drugs seem to have the ability to induce an autophagic cell death together with a minimal induction of apoptosis in melanoma cells. For example, JG-0314 , a microtubule poison, has been reported to have a cytotoxic effect on melanoma cells both in vitro and in vivo due to the induction of autophagy. Treatment of cells with autophagy inhibitors, such as chloroquine (CQ) or BafA1 demonstrated a moderate cytoprotection from JG-03-14. These seemingly contradictory findings were due to the fact that JG-03-14 induced apoptosis once autophagy had been blocked [61] . Chemotherapeutic agents, like JG-03-14, whose cytotoxic effect is due to induction of autophagy, would be especially suitable for anti-cancer therapy, because most of the cancer cells are anyway apoptosis-resistant. Consistent with the observation that the inhibition of autophagy induced apoptosis, Lakhter and colleagues newly showed that CQ 
promoted apoptosis in melanoma cells and inhibited melanoma tumor growth in vivo. The pro-apoptotic effect of CQ was due to its ability to stabilize a pro-apoptotic protein, PUMA, based on the observation that the transcription of PUMA remained unchanged upon CQ treatment and the protein stability was prolonged after exposure to a de novo protein synthesis inhibitor, cycloheximide in the presence of CQ. These authors also demonstrated that other agents affecting lysosomal $\mathrm{pH}$, such as BafA1 and the lysosomal cathepsin inhibitor ALLN, had no effect on PUMA protein stability. Moreover, the protease inhibitors leupeptin and lactacystin did not inhibit the degradation of PUMA. These data suggested that the apoptotic effect of CQ on melanoma cells via stabilization of PUMA was independent of lysosomal pathways [62]. A very interesting report on the photodynamic treatment of melanoma cells revealed apoptosis or autophagic cell death was dependent on the melanin content in melanoma cells. In this study, authors showed that 5-ALA, a photosensitizer used in photodynamic therapy, induced a p53dependent apoptosis in pigmented melanoma cells, whereas it caused an autophagydependent, caspase-independent cell death in non-pigmented melanoma cells [63] .

In the studies mentioned above, autophagy had been evaluated by Western Blot analysis to detect the lipidation of LC3 $[50-54,56,58-60,62]$ or the degradation of p62 $[50,56,61,62]$. Autophagosomes were visualized by the punctate pattern of either endogenous LC3 staining or overexpression of LC3 coupled with GFP (GFP-LC3) $[51,53,54,56,58,59,62]$ and MDC or acridine orange staining $[56,60,61]$. In some studies, electron microscopy was used to detect the double-membrane structure of autophagosomes $[51,54,56,58-60,61]$. Compared with the problem of evaluating the autophagy status in melanoma specimens from patients in vivo, measuring autophagy levels in melanoma cell lines would seem to offer more and better alternative experimental approaches, all of which should be applied in order to better characterize the autophagy status in vitro.

\section{Involvement of autophagy in melanoma therapies targeting an altered metabolic profile in tumor cells}


Targeted therapy against melanoma is undergoing intensive investigation to improve the outcome for patients. Recently, the development of BRAF inhibitors to target the V600E mutation has brought a new perspective in melanoma therapy, but drug resistance as an inevitable consequence still remains unsolved $[64,65]$. Depending on the genetic background, tumor cells may differ in their requirements for essential amino acids for their survival [66]. An altered metabolism is beneficial for the cancer cells; however, the demand for nutrients to support tumor growth is also increased in the neoplastic cells. In the center of the tumor, where the nutrient supply is often poor, autophagy as a catabolic process has been shown to be activated to ensure the survival $[66,67]$. To take advantage of this consideration, several studies have been carried out to find an alternative therapeutic approach targeting autophagy and altered metabolism in melanoma therapy.

In a study examining the response of melanoma cells towards deprivation of single amino acids, Sheen et al. have found that deprivation of leucine, but not other essential amino acids, resulted in apoptosis. Apoptotic cell death in melanoma cells could be partially rescued by the pan-caspase inhibitors Q-VD-OPH. Furthermore, the authors showed that leucine deprivation failed to induce autophagy in melanoma cells with hyperactivation of RAS-MEK pathway, although it induced autophagy in other tumor cell lines. In contrast to deprivation of all other amino acids leading to suppression of mTORC1 and activation of autophagy, mTORC1 activity was very poorly inhibited when leucine was deprived. Inhibiting autophagy by knocking down ATG1 or VPS34 (class III PI3K) expression in melanoma cells, or by applying the autophagy inhibitor, CQ, in tumor xenografts synergistically sensitized melanoma cells towards leucine-deprivationinduced cell death [68]. Another report by You et al., however, demonstrated that arginine deprivation in combination with the treatment with TRAIL induced an enhanced apoptotic cell death in melanoma cells, which are often argininosuccinate synthetase (ASS)-negative. Autophagy as a protective response to cellular stresses was induced when arginine was deprived in melanoma cells; however, treatment of cells with TRAIL activated caspase- 8 leading to the cleavage of Beclin 1 and ATG5, thereby inhibiting the induction of autophagy. Caspase- 8 inhibitor, which blocked the cleavage of Beclin 1 and 
ATG5, was able to reverse the enhanced cytotoxicity by TRAIL in combination with arginine deprivation, suggesting that the cleavage of Beclin 1 and ATG5 by TRAIL was at least one of the mechanisms leading to the effective cell killing seen with this combination therapy $[69]$.

Contrasting to the studies showing that inhibition of autophagy is beneficial for melanoma cell killing under amino-acid-deprived-condition, it has been reported that CQ induced a rapid melanoma cell death in serum-free medium independent of autophagy, because this effect was not recapitulated with autophagy inhibitors or by knocking down LC3 $[70]$. Furthermore, the authors showed that CQ-induced cytotoxicity of melanoma cells in the absence of serum is due to induction of apoptosis and necrosis resulting from mitochondrial depolarization. The action of CQ on melanoma cell death was not due to inhibition of autophagy, rather to an interaction with lysosomes. The lysosomal acidification inhibitor BafA1 prevented CQ-induced cell death under serumdeprived condition, just as the failure of blocking autophagy to enhance cell death. Thus, it seems that the lysosomal pathway, but not autophagy is essential in this process. Importantly, CQ potentiated the anti-tumor effect of calorie-restriction in mice [70], consistent with the previous findings that calorie-restriction increases the lifespan and reduces the incidence of numerous diseases, including cancer $[71,72]$.

\section{Hypoxia-induced autophagy in melanoma tumor progression}

Hypoxia as a common character of proliferating tumor cells has been shown to be able to induce autophagy in different cellular settings. For example, Naves et al. have recently shown that treatment with $\mathrm{CoCl}_{2}$, a hypoxia mimetic, induced autophagy in p53-mutated neuroblastoma cells. Pharmacological inhibition of autophagy enhanced apoptosis and cell death in this context $[73]$. In colon adenocarcinoma, autophagy was shown to be increased once disease progressed. Autophagy levels correlated to the expression of HIF$1 \alpha$, a transcription factor regulating hypoxia; and this hypoxia-driven autophagy is commonly associated with early invasion and severely dysplastic adenoma [74] . 
In melanoma, several recent reports showed the relationship between hypoxia, autophagy, and tumor progression. In a melanoma mouse model, in which B16F10 murine melanoma cells are injected subcutaneously, an accelerated proliferation and angiogenesis was demonstrated in beclin $1^{+/-}$mice compared with wild-type mice. This effect seen in beclin $1^{+/-}$mice was even more enhanced under hypoxic condition. Cells derived from beclin $1^{+/-}$mice expressed an elevated level of HIF-2 $\alpha$. These mice were compromised for hypoxia-induced autophagy, which was evidenced by increased autophagosome formation and induction of beclin 1 seen in the wild-type mice, suggesting melanoma tumors deficient for beclin 1 showed a pro-angiogenic phenotype associated with a hypoxic character $[75]$. Interestingly, Noman et al. have shown that hypoxia impaired tumor cells towards CTL-mediated lysis due to induction of autophagy. Furthermore, B16F10 melanoma cells deficient for beclin 1, exhibited growth inhibition and increased tumor apoptosis in vitro. Applying hydroxychloroquine in mice bearing B16F10 melanoma tumor cells dramatically reduced tumor growth $[76]$.

Another study investigating the regulation of (PEDF), a member of the serine protease inhibitor superfamily with anti-angiogenic and anti-metastatic activity in melanoma cells, revealed that hypoxia reduced PEDF levels via induction of autophagy and subsequent degradation of PEDF through an autophagic pathway. The results pointed out the potential contribution of hypoxia-induced autophagy for melanoma metastasis [77]. In human melanoma specimen, a link between autophagy and hypoxia has been shown by Sivridis et al. In their study to examine the expression of LC3, Beclin 1, HIF- $1 \alpha$, and HIF-2 $\alpha$ in 79 nodular melanomas, the authors found that high Beclin 1 and LC3 expression was correlated with hypoxia as indicated by HIF-1 $\alpha$ and HIF-2 $\alpha$ immunoreactivity $[78]$. However, it was mentioned that the staining pattern of LC3 was cytoplasmic diffuse rather than punctate $[78]$, raising the question whether a high level of LC3 expression could reflect an increased level of autophagy. These results show the contrasting opinions regarding the role of hypoxia-induced autophagy in tumor development and progression that might be, at least partially, due to the different model systems used in described studies. 


\section{Targeting autophagy in combination with immunotherapy in melanoma cells}

Alternative to the chemotherapeutic drugs which represent the mainstream of melanoma cancer therapy, immunotherapy is indispensable in enhancing the immune response of melanoma patients to improve the therapeutic outcome. Recently, ipilimumab, a novel, targeted human immunostimulatory monoclonal antibody that blocks CTLA-4, has shown survival benefit for metastatic melanoma patients [79]. As mentioned above, autophagy plays an important role in immunity and inflammation $[11,80]$. In the context of immunotherapy, the involvement of autophagy is becoming more and more evident. For instance, Tormo et al. have identified the dsRNA mimic polyinosinepolycytidylic acid as a novel autophagy inducer. Interestingly, naked polyinosinepolycytidylic acid activated a transient response of IFN and other antiviral stress response factors, while it induced a sustained stress response program once packed with polyethylene imine (PEI) for delivery of the dsRNA to the cells. The stress program included a rapid autophagic response and a strong induction of apoptosis thereafter. Moreover, autophagy induced in the early phase of the treatment is not a bystander or a protective process. It rather acts as a promoting factor in the induction of melanoma cytotoxicity, based on the observation, that wild-type mouse embryonic fibroblasts (MEFs) were more sensitive to polyinosine-polycytidylic-acid-induced cell death than the atg5 knockout counterpart [81].

A possible link between autophagy, apoptosis, and the immune response has been shown in melanoma cells treated with GA-DM, a natural triterpenoid derived from the medicinal mushroom Ganoderma lucidum [82, 83 ]. GA-DM induced an early autophagic response (3-6h) with increased expression of Beclin 1 and LC3 lipidation, followed by apoptosis in later stage $(24 \mathrm{~h})$ of the treatment. Blocking autophagy with 3MA at early time points resulted in cell death, which was remarkably increased beyond $12 \mathrm{~h}$ of GA-DM treatment regardless of 3-MA. These data indicate a cellular protection through autophagy in the early phase and that an excessive autophagic level could trigger a subsequent death event. These authors further proved that the eventual cell death was due to induction of caspase-dependent apoptosis. Importantly, autophagy induced in the early phase of the GA-DM treatment upregulated the HLA class II molecules as well as 
the expression of the lysosomal protein LAMP2 in melanoma cells. Western Blot analysis of HLA class II components indicated an activation of the HLA class II antigen processing machinery due to a significant increase in HLA-DR and HLA-DM proteins. Furthermore, blocking autophagy with 3-MA reduced CD4 $+\mathrm{T}$ cell response towards antigens presented by GA-DM treated melanoma cells $[83]$.

Downregulation or even loss of MHC class I antigen expression occurs very often in tumor cells, profoundly impeding antitumor immunity $[84,85]$. In contrast to a positive role of autophagy in MHC class II presentation $[86,87]$, a dual role of autophagy in the regulation of MHC class I antigen expression in B16 murine melanoma cells has been shown by $\mathrm{Li}$ et al. [88]. Whether autophagy is a positive or a negative regulator of the MHC class I antigen expression is dependent on IFN- $\gamma$ : in the presence of IFN- $\gamma$, autophagy upregulated the MHC class I antigen expression whereas it reduced MHC class I antigen expression in the absence of IFN- $\gamma$. The authors further showed an efficient CTL cytolysis at the early stage of B16 melanoma tumor progression, when IFN- $\gamma$ level is high, while it was largely impaired in the later stage of the tumor formation with immune escape of the tumor cells [88].

The proteins in the donor cells that are potentially accessible for cross-presentation are degraded either by proteasomal or autophagic pathways $[11]$, hence a reduction in autophagic levels in tumor cells would facilitate cross-presentation by host professional APCs. Surprisingly, Li et al. have recently shown that rapamycin and starvation, which induce autophagy, greatly increased the presentation of endogenous melanoma-specific antigen, gp100, by DCs with increased proliferation of CD8 + T cells. An opposite effect on antigen presentation has been shown in melanoma cells treated with 3-MA, which inhibits the early initiation of autophagosome formation. Interestingly, $\mathrm{NH}_{4} \mathrm{Cl}$, which prevents lysosome acidification and, thereby, the fusion of the autophagosome with the lysosome, augmented the cross-presentation by melanoma cells. These data indicated the biphasic role of autophagy in cross-presentation; in the early phase, autophagy is required for cross-presentation, whereas in the late phase it hinders. Down-regulation of the essential ATG proteins involved in the early phase of autophagy, such as Beclin 1 and 
ATG12 in melanoma cells, significantly reduced cross-presentation. Moreover, authors showed that isolated autophagosomes are the carriers of the protein antigens from the tumor cells, implying a potential role for autophagy in tumor immunotherapy [89] . A follow-up study by the same group further characterized Dribbles, the autophagosomeenriched, defective ribosomal products (DRiPs)-containing blebs and revealed the mechanism by which the enhanced efficacy of Dribbles as tumor vaccine in the B16F10 melanoma tumor model is achieved $[90]$.

\section{Concluding remarks}

As an ancient, cellular catabolic pathway, autophagy deserves to be investigated continuously and intensively in connection with the field of oncology, certainly including melanoma. Experimental data from increasing numbers of publications have given us confidence that autophagy is likely to represent an alternative target in melanoma cancer therapy in spite of the inconsistent and even contradictory findings reported in the literature (Fig. 2, Table 1). One important step, which is unavoidable, is to evaluate the autophagic levels in melanoma in vivo. The current strategy is either to detect the expression of autophagy-related marker proteins by immunohistology or to detect the double-membrane autophagosomes in melanoma specimens by electron microscopy. However, the reagents and the staining procedures should be standardized to avoid technical artifacts. Additionally, the cohort of melanoma patients with clinical annotations enrolled on the study should be sufficiently large to allow definitive conclusions. Similar to measuring the autophagic flux in cell lines in vitro, a kinetic analysis of e.g. autophagosome formation should be carried out using melanoma three dimensional cultures mimicking, at least to a certain extent, melanoma tumors in vivo.

Given that mTOR and the PI3K have pleiotropic functions, the agents used to target these pathways are potent, but not specific enough in regulating autophagy. Unfortunately, but not surprisingly, the statement that inhibition of autophagy facilitates the cytotoxic effect of a given compound is largely based on studies with these inhibitors. Whether the increased efficacy of the cytotoxic compounds in combination with these 
inhibitors is indeed due to inhibition of autophagy or other mechanisms, needs to be clarified. Genetic manipulation of ATG proteins should be considered as an alternative approach in addition to treatments with these inhibitors.

The question whether autophagy supports pro- or anti-tumor formation in melanoma pathology still remains unsolved. Future studies to characterize the autophagic levels as well as the expression of ATG proteins in melanoma varying from primary to metastatic stage will hopefully enlighten us with respect to the role of autophagy in disease progression. In addition, such a role for melanoma cancer stem cells needs also to be considered in order to reduce the reoccurrence after conventional therapy, since autophagy is required to maintain the stemness of skin-derived precursors (SKPs) [91] . Altogether, the road to unveiling the role of autophagy in melanoma cancer therapy is rugged, but hopeful. Basic researchers should bring strong evidence for the importance of autophagy and should collaborate closely with dermatologists and other clinicians to realize the potential of this field for effective anti-melanoma treatment.

\section{Acknowledgements}

We apologize to all the researchers whose work was not cited or discussed in this review due to space limitations. Work at the Simon's laboratory is supported by Swiss National Science Foundation (grant No. 310030_146181), Swiss Cancer League, and Stiftung zur Krebsbekämpfung, Zurich. 


\section{References}

[1] Reggiori F, Shintani T, Nair U, Klionsky DJ. Atg9 cycles between mitochondria and the pre-autophagosomal structure in yeasts. Autophagy 2005; $1(2): 101-9$.

[2] Hayashi-Nishino M, Fujita N, Noda T, Yamaguchi A, Yoshimori T, Yamamoto A. A subdomain of the endoplasmic reticulum forms a cradle for autophagosome formation. Nat Cell Biol 2009; 11(12):1433-7.

[3] Hayashi-Nishino M, Fujita N, Noda T, Yamaguchi A, Yoshimori T, Yamamoto A. Electron tomography reveals the endoplasmic reticulum as a membrane source for autophagosome formation. Autophagy 2010; 6(2):301-3.

[4] Cuervo AM. Autophagy in sickness and in health. Trends Cell Biol 2004; $14(2): 70-77$.

[5] Mizushima N, Levine B, Cuervo AM, Klionsky DJ. Autophagy fights disease through cellular self-digestion. Nature 2008; 451(7182):1069-75.

[6] Shintani T, Klionsky DJ. Autophagy in health and disease: a double-edged sword. Science 2004; 306(5698):990-5.

[7] Levine B, Klionsky DJ. Development by self-digestion: molecular mechanisms and biological functions of autophagy. Dev Cell 2004; 6(4):463-77.

[8] Levine B, Yuan J. Autophagy in cell death: an innocent convict? J Clin Invest 2005; 115(10):2679-88.

[9] Yousefi S, Simon HU. Apoptosis regulation by autophagy gene 5. Crit Rev Oncol Hematol 2007; 63(3):241-4.

[10] Wu YT, Tan HL, Shui G, Bauvy C, Huang Q, Wenk MR, et al. Dual role of 3methyladenine in modulation of autophagy via different temporal patterns of inhibition on class I and III phosphoinositide 3-kinase. J Biol Chem 2010; 285(14):10850-61.

[11] Levine B, Mizushima N, Virgin HW. Autophagy in immunity and inflammation. Nature 2011; 469(7330):323-35.

[12] Wirawan E, Vanden Berghe T, Lippens S, Agostinis P, Vandenabeele P. Autophagy: for better or for worse. Cell Res 2012; 22(1):43-61. 
[13] Yousefi S, Perozzo R, Schmid I, Ziemiecki A, Schaffner T, Scapozza L, et al. Calpain-mediated cleavage of Atg5 switches autophagy to apoptosis. Nat Cell Biol 2006; 8(10):1124 - 32.

[14] Pattingre S, Tassa A, Qu X, Garuti R, Liang XH, Mizushima N, et al. Bcl-2 antiapoptotic proteins inhibit Beclin 1-dependent autophagy. Cell 2005; 122(6):927-39.

[15] Chen ZH, Lam HC, Jin Y, Kim HP, Cao J, Lee SJ, et al. Autophagy protein microtubule-associated protein 1 light chain-3B (LC3B) activates extrinsic apoptosis during cigarette smoke-induced emphysema. Proc Natl Acad Sci USA $2010 ; 107(44): 18880-5$.

[16] Kroemer G, Mariño G, Levine B. Autophagy and the integrated stress response. Mol Cell 2010; 40(2):280-93.

[17] Mizushima N, Levine B. Autophagy in mammalian development and differentiation. Nat Cell Biol 2010;12(9):823-30.

[18] Rubinsztein DC, Mariño G, Kroemer G. Autophagy and aging. Cell 2011; 146(5):682-95.

[19] Rubinsztein DC, Codogno P, Levine B. Autophagy modulation as a potential therapeutic target for diverse diseases. Nat Rev Drug Discov 2012; 11(9):70930.

[20] Rubinsztein DC. The roles of intracellular protein-degradation pathways in neurodegeneration. Nature 2006; 443(7113):780-6.

[21] Martinez-Vicente M, Cuervo AM. Autophagy and neurodegeneration: when the cleaning crew goes on strike. Lancet Neurol 2007; 6(4):352-61.

[22] Tannous P, Zhu H, Johnstone JL, Shelton JM, Rajasekaran NS, Benjamin IJ, et al. Autophagy is an adaptive response in desmin-related cardiomyopathy. Proc Natl Acad Sci USA 2008; 105(28):9745-50.

[23] Kirshenbaum LA. Regulation of autophagy in the heart in health and disease. J Cardiovasc Pharmacol 2012; 60(2):109.

[24] He C, Bassik MC, Moresi V, Sun K, Wei Y, Zou Z, et al. Exercise-induced BCL2-regulated autophagy is required for muscle glucose homeostasis. Nature 2012; 481(7382):511-5. 
[25] Yang L, Li P, Fu S, Calay ES, Hotamisligil GS. Defective hepatic autophagy in obesity promotes ER stress and causes insulin resistance. Cell Metab 2010; 11(6):467-78.

[26] Singh R, Xiang Y, Wang Y, Baikati K, Cuervo AM, Luu YK, et al. Autophagy regulates adipose mass and differentiation in mice. J Clin Invest 2009; 119(11):3329-39.

[27] Chen ZH, Kim HP, Sciurba FC, Lee SJ, Feghali-Bostwick C, Stolz DB, et al. Egr-1 regulates autophagy in cigarette smoke-induced chronic obstructive pulmonary disease. PLoS One 2008; 3(10):e3316.

[28] Luciani A, Villella VR, Esposito S, Brunetti-Pierri N, Medina D, Settembre C, et al. Defective CFTR induces aggresome formation and lung inflammation in cystic fibrosis through ROS-mediated autophagy inhibition. Nat Cell Biol 2010; 12(9):863-75.

[29] Patel AS, Lin L, Geyer A, Haspel JA, An CH, Cao J, et al. Autophagy in idiopathic pulmonary fibrosis. PLoS One 2012; 7(7):e41394.

[30] Yousefi S, Simon HU. Autophagy in cancer and chemotherapy. Results Probl Cell Differ 2009; 49:183-90.

[31] White E. Deconvoluting the context-dependent role for autophagy in cancer. Nat Rev Cancer 2012; 12(6):401-10.

[32] Choi AM, Ryter SW, Levine B. Autophagy in human health and disease. N Engl J Med 2013; 368(7):651-62.

[33] Gozuacik D, Kimchi A. Autophagy as a cell death and tumor suppressor mechanism. Oncogene 2004; 23(16):2891-906.

[34] Tschan MP, Simon HU. The role of autophagy in anticancer therapy: promises and uncertainties. J Intern Med 2010; 268(5):410-8.

[35] Young AR, Narita M, Ferreira M, Kirschner K, Sadaie M, Darot JF, et al. Autophagy mediates the mitotic senescence transition. Genes Dev 2009; 23(7):798-803.

[36] Liang XH, Jackson S, Seaman M, Brown K, Kempkes B, Hibshoosh H, et al. Induction of autophagy and inhibition of tumorigenesis by Beclin1. Nature 1999; 420(6762):672-6. 
[37] Saito H, Inazawa J, Saito S, Kasumi F, Koi S, Sagae S, et al. Detailed deletion mapping of chromosome $17 \mathrm{q}$ in ovarian and breast cancers: 2-cM region on $17 q 21.3$ often and commonly deleted in tumors. Cancer Res 1993; 53(14):3382-5.

[38] Gao X, Zacharek A, Salkowski A, Grignon DJ, Sakr W, Porter AT, et al. Loss of heterozygosity of the BRCA1 and other loci on chromosome $17 \mathrm{q}$ in human prostate cancer. Cancer Res 1995; 55(5):1002-5.

[39] Liang C, Feng P, Ku B, Dotan I, Canaani D, Oh BH, et al. Autophagic and tumor suppressor activity of a novel Beclin1-binding protein UVRAG. Nat Cell Biol 2006; 8(7):688-99.

[40] Takahashi Y, Coppola D, Matsushita N, Cualing HD, Sun M, Sato Y, et al. Bif-1 interacts with Beclin 1 through UVRAG and regulates autophagy and tumorigenesis. Nat Cell Biol 2007; 9(10):1142-51.

[41] Takamura A, Komatsu M, Hara T, Sakamoto A, Kishi C, Waguri S, et al. Autophagy-deficient mice develop multiple liver tumors. Genes Dev 2011; 25(8):795-800.

[42] Guertin DA, Sabatini DM. Defining the role of mTOR in cancer. Cancer Cell 2007; 12(1):9-22.

[43] Díaz-Troya S, Pérez-Pérez ME, Florencio FJ, Crespo JL. The role of TOR in autophagy regulation from yeast to plants and mammals. Autophagy 2008; $4(7): 851-65$.

[44] Klionsky DJ, Abdalla FC, Abeliovich H, Abraham RT, Acevedo-Arozena A, Adeli K, et al. Guidelines for the use and interpretation of assays for monitoring autophagy. Autophagy 2012; 8(4):445-544.

[45] Lazova R, Klump V, Pawelek J. Autophagy in cutaneous malignant melanoma. J Cutan Pathol 2010; 37(2):256-68.

[46] Miracco C, Cevenini G, Franchi A, Luzi P, Cosci E, Mourmouras V et al. Beclin 1 and LC3 autophagic gene expression in cutaneous melanocytic lesions. Hum Pathol 2010; 41(4):503-12. 
[47] Hara Y, Nakamura M. Overexpression of autophagy-related beclin-1 in advanced malignant melanoma and its low expression in melanoma-in-situ. Eur J Dermatol 2012; 22(1):128-9.

[48] Sivridis E, Koukourakis MI, Mendrinos SE, Karpouzis A, Fiska A, Kouskoukis C, et al. Beclin-1 and LC3A expression in cutaneous malignant melanomas: a biphasic survival pattern for beclin-1. Melanoma Res 2011; 21(3):188-95.

[49] Ma XH, Piao S, Wang D, McAfee QW, Nathanson KL, Lum JJ, et al. Measurements of tumor cell autophagy predict invasiveness, resistance to chemotherapy, and survival in melanoma. Clin Cancer Res 2011; 17(10):347889.

[50] Marino ML, Fais S, Djavaheri-Mergny M, Villa A, Meschini S, Lozupone F, et al. Proton pump inhibition induces autophagy as a survival mechanism following oxidative stress in human melanoma cells. Cell Death Dis 2010; $1: \mathrm{e} 87$.

[51] Hammerová J, Uldrijan S, Táborská E, Vaculová AH, Slaninová I. Necroptosis modulated by autophagy is a predominant form of melanoma cell death induced by sanguilutine. Biol Chem 2012; 393(7):647-58.

[52] Düchler M. G-quadruplexes: targets and tools in anticancer drug design. J Durg Target 2012; 20(5):389-400.

[53] Orlotti NI, Cimino-Reale G, Borghini E, Pennati M, Sissi C, Perrone F, et al. Autophagy acts as a safeguard mechanism against G-quadruplex ligandmediated DNA damage. Autophagy. 2012; 8(8):1185-96.

[54] Marino ML, Pellegrini P, Di Lernia G, Djavaheri-Mergny M, Brnjic S, Zhang $\mathrm{X}$, et al. Autophagy is a protective mechanism for human melanoma cells under acidic stress. J Biol Chem 2012; 287(36):30664-76.

[55] Dominy JE Jr, Simmons CR, Hirschberger LL, Hwang J, Coloso RM, Stipanuk MH. Discovery and characterization of a second mammalian thiol dioxygenase, cysteamine dioxygenase. J Biol Chem 2007; 282(35):25189-98.

[56] Wan XM, Zheng F, Zhang L, Miao YY, Man N, Wen LP. Autophagymediated chemosensitization by cysteamine in cancer cells. Int J Cancer 2011; 129(5):1087-95. 
[57] Bailey CJ, Turner RC. Metformin. N Engl J Med 1996; 334(9):574-9.

[58] Tomic T, Botton T, Cerezo M, Robert G, Luciano F, Puissant A, et al. Metformin inhibits melanoma development through autophagy and apoptosis mechanisms. Cell Death Dis 2011; 2:e199.

[59] Nicolau-Galmés F, Asumendi A, Alonso-Tejerina E, Pérez-Yarza G, Jangi SM, Gardeazabal J, et al. Terfenadine induces apoptosis and autophagy in melanoma cells through ROS-dependent and -independent mechanisms. Apoptosis 2011; 16(12):1253-67.

[60] Liu B, Cheng Y, Zhang B, Bian HJ, Bao JK. Polygonatum cyrtonema lectin induces apoptosis and autophagy in human melanoma A375 cells through a mitochondria-mediated ROS-p38-p53 pathway. Cancer Lett 2009; 275(1):5460 .

[61] Biggers JW, Nguyen T, Di X, Gupton JT, Henderson SC, Emery SM, et al. Autophagy, cell death and sustained senescence arrest in B16/F10 melanoma cells and HCT-116 colon carcinoma cells in response to the novel microtubule poison, JG-03-14. Cancer Thermother Pharmacol 2013; 71(2):441-55.

[62] Lakhter AJ, Sahu RP, Sun Y, Kaufmann WK, Androphy EJ, Travers JB, et al. Chloroquine Promotes Apoptosis in Melanoma Cells by Inhibiting BH3 domain Mediated PUMA Degradation. J Invest Dermatol 2013; doi: $10.1038 /$ jid. 2013.56

[63] Sparsa A, Bellaton S, Naves T, Jauberteau MO, Bonnetblanc JM, Sol V, et al. Photodynamic treatment induces cell death by apoptosis or autophagy depending on the melanin content in two B16 melanoma cell lines. Oncol Rep 2013; 29(3):1196-200.

[64] Flaherty KT, Infante JR, Daud A, Gonzalez R, Kefford RF, Sosman J, et al. Combined BRAF and MEK inhibition in melanoma with BRAF V600 mutations. N Engl J Med 2012; 367(18):1694-703.

[65] Sosman JA, Kim KB, Schuchter L, Gonzalez R, Pavlick AC, Weber JS, et al. Survival in BRAF V600-mutant advanced melanoma treated with vemurafenib. N Engl J Med 2012; 366(8):707-14. 
[66] Cheong H, Lindsten T, Thompson CB. Autophagy and ammonia. Autophagy 2012; 8(1):122-3.

[67] Ma XH, Piao S, Wang D, McAfee QW, Nathanson KL, Lum JJ, et al. Measurements of tumor cell autophagy predict invasiveness, resistance to chemotherapy, and survival in melanoma. Clin Cancer Res 2011; 17(10):347889.

[68] Sheen JH, Zoncu R, Kim D, Sabatini DM. Defective regulation of autophagy upon leucine deprivation reveals a targetable liability of human melanoma cells in vitro and in vivo. Cancer Cell 2011; 19(5):613-28.

[69] You M, Savaraj N, Kuo MT, Wangpaichitr M, Varona-Santos J, Wu C, et al. TRAIL induces autophagic protein cleavage through caspase activation in melanoma cell lines under arginine deprivation. Mol Cell Biochem 2013; 374(1-2):181-90.

[70] Harhaji-Trajkovic L, Arsikin K, Kravic-Stevovic T, Petricevic S, Tovilovic G, Pantovic A, et al. Chloroquine-mediated lysosomal dysfunction enhances the anticancer effect of nutrient deprivation. Pharm Res 2012; 29(8):2249-63.

[71] Kennedy BK, Steffen KK, Kaeberlein M. Ruminations on dietary restriction and aging. Cell Mol Life Sci 2007; 64(11):1323-8.

[72] Omodei D, Fontana L. Calorie restriction and prevention of age-associated chronic disease. FEBS Lett 2011; 585(11):1537-42.

[73] Naves T, Jawhari S, Jauberteau MO, Ratinaud MH, Verdier M. Autophagy takes place in mutated p53 neuroblastoma cells in response to hypoxia mimetic $\mathrm{CoCl}_{2}$. Biochem Pharmacol 2013; 85(8):1153-61.

[74] Giatromanolaki A, Koukourakis MI, Koutsopoulos AV, Harris AL, Gatter K, Sivridis E. Autophagy and hypoxia in colonic adenomas related to aggressive features. Colorectal Dis 2013; 15(5):e223-30.

[75] Lee SJ, Kim HP, Jin Y, Choi AM, Ryter SW. Beclin 1 deficiency is associated with increased hypoxia-induced angiogenesis. Autophagy 2011; 7(8):829-39.

[76] Noman MZ, Janji B, Kaminska B, Van Moer K, Pierson S, Przanowski P, et al. Blocking hypoxia-induced autophagy in tumors restores cytotoxic T-cell activity and promotes regression. Cancer Res 2011; 71(18):5976-86. 
[77] Fernández-Barral A, Orgaz JL, Gomez V, del Peso L, Calzada MJ, Jiménez B. Hypoxia negatively regulates antimetastatic PEDF in melanoma cells by a hypoxia inducible factor-independent, autophagy dependent mechanism. PLoS One 2012; 7(3):e32989.

[78] Sivridis E, Koukourakis MI, Mendrinos SE, Karpouzis A, Fiska A, Kouskoukis C, et al. Beclin-1 and LC3A expression in cutaneous malignant melanomas: a biphasic survival pattern for beclin-1. Melanoma Res 2011;21(3):188-95.

[79] Boasberg P, Hamid O, O'Day S. Ipilimumab: unleashing the power of the immune system through CTLA-4 blockade. Semin Oncol 2010;37(5):440-9.

[80] Levine B, Deretic V. Unveiling the roles of autophagy in innate and adaptive immunity. Nat Rev Immunol 2007; 7(10):767-77.

[81] Tormo D, Checińska A, Alonso-Curbelo D, Pérez-Guijarro E, Cañón E, Riveiro-Falkenbach E, et al. Targeted activation of innate immunity for therapeutic induction of autophagy and apoptosis in melanoma cells. Cancer Cell 2009; 16(2):103-14.

[82] Boh B, Berovic M, Zhang J, Zhi-Bin L. Ganoderma lucidum and its pharmaceutically active compounds. Biotechnol Annu Rev 2007; 13:265-301.

[83] Hossain A, Radwan FF, Doonan BP, God JM, Zhang L, Bell PD, et al. A possible cross-talk between autophagy and apoptosis in generating an immune response in melanoma. Apoptosis 2012; 17(10):1066-78.

[84] McDougall CJ, Ngoi SS, Goldman IS, Godwin T, Felix J, DeCosse JJ, et al. Reduced expression of HLA class I and II antigens in colon cancer. Cancer Res 1990; 50(24):8023-7.

[85] Hicklin DJ, Marincola FM, Ferrone S. HLA class I antigen downregulation in human cancers: T-cell immunotherapy revives an old story. Mol Med Today 1999; 5(4):178-86.

[86] Levine B, Deretic V. Unveiling the roles of autophagy in innate and adaptive immunity. Nat Rev Immunol 2007;7(10):767-77.

[87] Münz C. Antigen processing for MHC class II presentation via autophagy. Front Immunol 2012;3:9. 
[88] Li B, Lei Z, Lichty BD, Li D, Zhang GM, Feng ZH, et al. Autophagy facilitates major histocompatibility complex class I expression induced by IFN$\gamma$ in B16 melanoma cells. Cancer Immunol Immunother 2010;59(2):313-21.

[89] Li Y, Wang LX, Yang G, Hao F, Urba WJ, Hu HM. Efficient crosspresentation depends on autophagy in tumor cells. Cancer Res 2008; 68(17):6889-95.

[90] Li Y, Wang LX, Pang P, Cui Z, Aung S, Haley D, et al. Tumor-derived autophagosome vaccine: mechanism of cross-presentation and therapeutic efficacy. Clin Cancer Res 2011; 17(22):7047-57.

[91] Salemi S, Yousefi S, Constantinescu MA, Fey MF, Simon HU. Autophagy is required for self-renewal and differentiation of adult human stem cells. Cell Res 2012; 22(2):432-5. 


\section{Figure and table legends:}

Fig. 1. The autophagic pathway. Autophagy can be divided into 3 main steps: Initiation, elongation, and degradation steps. Autophagy is induced by signaling cascades involving mTOR, class I and class III PI3K (VPS34). The mTOR inhibits autophagy through inhibiting ATG1/ULK1. Class I PI3K activates mTOR, therefore inhibits autophagy. During the initiation step of autophagy a large protein complex, including Beclin 1, the class III PI3K, VPS34, ATG14L, and p150, is formed. Activation of this complex generates PI3P, facilitating the nucleation of autophagosomal membrane. Several interacting proteins which participate in this complex include positive factors, such as UVRAG, Bif-1, and AMBRA1, and the negative factor Rubicon. In the elongation step, two distinct ubiquitin-like conjugation systems are involved. The ubiquitin-like ATG12 is conjugated to ATG5 by the E1-like ATG7 and the E2-like ATG10 enzymes. The conjugated ATG5-ATG12 then forms a complex with ATG16L, which is required in the elongation of the autophagosome membrane. The main player in the second conjugation system is LC3, which is cleaved by the protease ATG4 to generate the LC3-I form. LC3-I is decorated with phosphatidylethanolamine (PE) with the help of E1-like ATG7 and E2like ATG3 forming the LC3-II form. Finally, in the degradation step, engulfed contents are degraded after the fusion of autophagosome with a lysosome. Chemical compounds which target the different regulators in the autophagic pathway are also indicated in the schema.

Fig. 2. Targeting autophagy for improved melanoma cancer therapy. Depending on the autophagy level in melanoma and the contribution of autophagy to eliciting tumor cell death, either an inhibition or an induction of autophagy can be considered in combination with other conventional anti-melanoma therapies. These ideas are presented in simplified form in this schema.

Table 1. Effects of autophagy-regulating drugs on chemotherapy-induced melanoma cell death. 